\title{
Melitocoria de Zygia racemosa (Ducke) Barneby \& Grimes por Melipona seminigra merrillae Cockerell, 1919 y Melipona compressipes manaosensis Schwarz, 1932 (Hymenoptera, Meliponina) en la Amazonía Central, Brasil
}

\author{
Christinny Giselly BACELAR-LIMA ', Delci da Costa Brito FREIRE ${ }^{2}$, Alexandre COLETTO-SILVA ${ }^{3}$, \\ Klilton Barbosa da COSTA ${ }^{4}$, Jonilson Paulo Barros LARAY ${ }^{4}$, Hélio Conceição VILAS-BOAS ${ }^{4}$, Gislene \\ Almeida CARVALHO-ZILSE ${ }^{4}$
}

\section{RESUMO}

Durante el periodo de enero a abril de 2005 fueron encontradas semillas de Zygia racemosa, incorporadas al geoprópolis, en el interior de abejas sin aguijón en dos meliponarios experimentales en Manaus - AM. Fue observada intensa actividad de las obreras de $M$. seminigra merrillae y $M$. compressipes manaosensis llegando del campo con semillas fijas en las corbículas adheridas con resina y/o saliendo de las colonias con las semillas atrapadas en las mandíbulas. Ochenta semillas fueron recogidas en el interior de las colonias (mezcladas al geoprópolis en los basureros y fisuras) y también en el exterior, cerca de la entrada de las colonias, como resultado de la caída de esas semillas de las corbículas durante el vuelo de las obreras. Las semillas fueron plantadas en semilleros para la producción de esquejes y posterior identificación de la especie vegetal. Paralelamente se realizó el rastreo en campo en un radio de aproximadamente $3 \mathrm{Km}$ para la confirmación de la dispersión de las semillas, observaciones de comportamiento de las abejas forrajeando y recolección de semillas. Un total de 170 plántulas de $Z$. racemosa fueron encontradas en los alrededores del meliponario del GPA-INPA y 160 en el meliponario Vale Verde. Esos resultados indican que tanto M. compressipes manaosensis como M. seminigra merrillae recolectan y dispersan las semillas de $Z$. racemosa.

\section{PALABRAS-CLAVE}

Semillas, dispersión, meliponíneos, meliponicultura, angelim rajado.

\section{Melitocory of Zygia racemosa (Ducke) Barneby \& Grimes by Melipona seminigra merrillae Cockerell, 1919 and Melipona compressipes manaosensis Schwarz, 1932 (Hymenoptera, Meliponina) in Central Amazon, Brazil}

\begin{abstract}
During the period from January to April 2005 seeds of Zygia racemosa were found incorporated in the nest structures, inside the colonies of stingless bee in two experimental meliponaries in Manaus - Am. It was registered an intense activity of the bee-workers of Melipona seminigra merrillae and M. compressipes manaosensis arriving from the field with seeds set in the corbiculae adhered with resin and/or leaving the colonies with the seeds clipped in their jaws. Eighty seeds were collected inside the colonies (mixed with the batume in the litter andlor cracks), and also outside them, near the entrance of the colony, as a result of the falling of corbiculae during the flight of the bee-workers. These seeds were planted in seedbeds for seedling production and posterior identification of the botanical species. As a way to confirm the scattering of the seeds, searches were done in the field (in an area of approximately $3 \mathrm{~km}$ of extension), as well as observations of the collection of the seeds made by the bees. A total of 170 seedlings of Z. racemosa were found in the outskirts of the GPA-INPA meliponary and 160 in the Vale Verde meliponary. The results indicate that M. compressipes and M. seminigra collect and scatter the seeds of Z. racemosa.
\end{abstract}

\section{KEY WORDS}

Seeds, scattering, meliponines, meliponiculture, angelim-rajado.

${ }^{1}$ Instituto Nacional de Pesquisas da Amazônia (INPA), Coordenação de Pesquisas em Botânica, ACF Vieiralves, Caixa Postal 4167, CEP 69053-971, Manaus, Amazonas, Brasil. E-mail: christinnyg@yahoo.com.br

${ }^{2}$ Secretaria Municipal de Educação, Gerência Distrital Zona Rural. Rua Rio Içá 850, Vieiralves, Manaus, Amazonas, Brasil;

${ }^{3}$ INPA, Coordenação de Pesquisas em Ciências Agronômicas (CPCA), ACF Vieiralves, Caixa Postal 4167, CEP 69053-971, Manaus, Amazonas, Brasil;

${ }^{4}$ INPA, CPCA, Grupo de Pesquisas em Abelhas, Av. André Araújo, 2936, Petrópolis, CEP 69083-000, Caixa Postal 478, Manaus, Amazonas, Brasil. 


\section{INTRODUCCIÓN}

Las abejas sin aguijón conocidas por meliponíneos desempeñan una función importante en el mantenimiento de diferentes ecosistemas, siendo consideradas como las principales responsables por la polinización de los árboles dependiendo del área geográfica considerada (Kerr et al., 1999). Además de este servicio prestado a la naturaleza, los meliponíneos también suministran miel y polen de sabores inigualables, apreciados tanto en el mercado brasileño como en el internacional. Las culturas tradicionales además de utilizar la miel y el polen en la alimentación, también están aprendiendo a utilizar esas abejas de manera racional y esto ha contribuido para el crecimiento de la meliponicultura en el Estado del Amazonas (Kerr et al., 2001), siguiendo el ejemplo de otras iniciativas en diferentes regiones brasileñas.

Esas abejas presentan una gran diversidad de comportamientos y suman aproximadamente 300 especies ya descritas en la literatura siendo 192 presentes en Brasil (Silveira et al., 2002). En su hábitat natural, ellas utilizan principalmente los huecos de los árboles para la construcción de sus nidos. Varios tipos de substratos naturales son utilizados por las diferentes especies de abejas para la construcción de las entradas y de la estructura interna de los nidos así como también de espacios y de control de la ventilación y de la temperatura de la colonia (Ihering, 1903a y b; Nogueira-Neto, 1997). Coelho de Seabra (1799) apud Nogueira-Neto (1997) discurrió sobre los substratos utilizados por abejas en la arquitectura de los nidos cuando relató la presencia de trozos de madera mezclados con la espesa cáscara del nido de las abejas “irapuá” (Trigona spinipes Fabricius, 1793). Roubik (1983a) describe que la Trigona nigerrima Cresson, 1878 y la Trigona dallatorreana Friese, 1900 construyen sus nidos a partir de una mezcla de fibras vegetales recolectadas en la madera podrida con resinas. En el caso de las resinas vegetales, estas son muy utilizadas por diferentes grupos de abejas, entre ellas Megachilidae, Euglossina y Meliponina. Los meliponíneos, en especial, utilizan las resinas principalmente para el molde de la arquitectura de sus nidos. Además de servir como material de construcción, las resinas pueden funcionar como defensa contra los microorganismos o enemigos naturales más grandes y también como localización del nido (Michener, 1974; Roubik, 1983a y b; Roubik, 1989).

Cuando recolectan resinas, las abejas pueden traer algunas semillas asociadas (Absy \& Kerr, 1977; Roubik, 1983b y 1989; Garcia et al., 1992; Wallace \& Trueman, 1995; Oliveira, 2001). Roubik (1983b y 1989) verificó que Melipona fuliginosa Lepeletier, 1836 utiliza, en la entrada y en el molde de gran parte de la estructura de la arquitectura del nido, semillas y resinas de los frutos de Vismia sp. Vand., 1788 (Clusiaceae).

Wallace \& Trueman (1995) en Austrália, demostraron que obreras de Trigona carbonaria Smith, 1854 recolectan semillas con resinas de Corymbia torelliana (F. Muell.) K.D. Hill \& L.A.S.
Johnson (1995). Estos autores confirmaron que T. carbonaria es capaz de dispersar semillas de C. torelliana a distancias de más de 300 metros.

Absy \& Kerr (1977) al estudiar la carga transportada por 267 abejas de Melipona seminigra merrillae Cockerell, 1919 verificaron que 104 (39\%) transportaban resinas del fruto de Vismia sp. (conocida popularmente como lacre). Fue el primer registro en la literatura donde abejas del género Melipona podrían estar contribuyendo para la dispersión de semillas de plantas. Mientras, Garcia et al. (1992) afirmam que las semillas no son de Vismia sp. pero si Coussapoa asperifolia Trécul ssp. magnifolia (Trécul) Akkermans \& Berg, 1982. En su trabajo registraron cinco especies de abejas (Melipona lateralis Erichson, 1840, $M$. seminigra merrillae, M. seminigra seminigra Friese, 1903, Trigona williana Friese, 1900 y M. rufiventris Lepeletier, 1836) recolectando epicarpio de los frutos de $C$. asperifolia junto con las semillas. Aunque los registros existentes en la literatura señalen apenas dos especies vegetales dispersadas por meliponíneos (Coussapoa sp. y Vismia sp.), este trabajo presenta una especie más, el angelim rajado - Zygia racemosa (Ducke) Barneby \& J.W. Grimes, 1997. Además de aumentar esa lista de especies vegetales dispersadas por abejas sin aguijón, en especial $M$. seminigra merrillae y $M$. compressipes manaosensis Schwarz, 1932, este trabajo también contribuye para el aumento del conocimiento sobre esas abejas en la Amazonía.

Zygia racemosa, popularmente conocida como "angelim rajado", es una especie forestal de gran importancia en la industria de la madera por su dureza. La madera se aprovecha en la construcción en general, carpintería de lujo, tabiques de pisos (parqué), durmientes, mangos de servicios, objetos de adorno y otros (Fonseca et al., 1991; Ferreira et al., 2001). La planta típicamente tropical pertenece a la familia Leguminosae: Mimosoideae, formando parte del dosel que según Lima-Filho et al. (2004) se encuentra en el estrato superior (25-30 metros de altura). De acuerdo con Alencar (1998), la especie es perenne, florece entre julio y octubre y fructifica entre septiembre y febrero. Sus frutos son secos del tipo folículo (Fig. 1), policárpicos y decientes cuando maduros (Ribeiro et al., 1999). En un sondeo preliminar sobre los medios de propagación de las especies madereras en la región de Manaus, se constató que el "angelim" obtiene su dispersión por animales, así como la mayoría de las especies forestales (Ferraz et al., 2004). Actualmente el "angelim rajado" se encuentra en la lista de las especies protegidas por el Instituto Brasileño de Protección al Medio Ambiente y Recursos Naturales Renovables - IBAMA debido a la explotación de su madera, siendo clasificada como especie vulnerable a la extinción. A mediados de los años 90 ya existía producción de madera en cantidades limitadas en los estados de Pará y Amazonas (Fonseca et al., 1991).

El objetivo de este trabajo ha sido el de estudiar la relación entre comportamientos de recolecta y dispersión de semillas de 
Z. racemosa por abejas sin aguijón, a partir de observaciones preliminares indicando la presencia de semillas de esa especie en el interior y exterior de colmenas racionales.

\section{MATERIAL Y MÉTODOS}

Fueron utilizadas colmenas de M. seminigra merrillae y $M$. compressipes manaosensis del meliponário del GPA-INPA (Sede, Manaus-AM, 03 05.838' S y 059 59.103' W) y del meliponário Vale Verde (Km 08, carretera BR-174, Manaus$\mathrm{AM}-02^{\circ} 54.866^{\prime} \mathrm{S}$ y $\left.060^{\circ} 02.881^{\prime} \mathrm{W}\right)$ para la observación y para la recolección de semillas presentes interna y externamente en las colonias. Las colmenas utilizadas en este experimento estaban en cajas racionales dispuestas tanto en sustentáculos individuales como en repisas colectivas. Las observaciones fueron realizadas desde enero hasta abril de 2005.

El área de la sede del INPA, considerado un fragmento forestal urbano, es un área verde preservada parcialmente con la introducción de algunas especies de importancia económica, ecológica y ornamental (Ferreira \& Ramos 1993). La vegetación del lugar se caracteriza como secundaria (Gentry, 1978) con la mayor parte de las especies constituidas por representantes de floresta primaria (Prance, 1975). La sede del INPA con 14 ha está separada por una avenida, de un área con 540 ha de floresta perteneciente a la Universidad Federal de Amazonas (UFAM).

El meliponario Vale Verde se ubica en una zona que presenta varias culturas de plantas, destacándose la pupunha - Bactris gasipaes Khunt, 1816 y el camu-camu - Myrciaria dubia (Kunth) McVaugh, 1963. En este meliponario, las colmenas se encontraban a una distancia de aproximadamente 200 metros de la vegetación natural de floresta.

El clima de la región se caracteriza como "Afi" en la clasificación de Köpen, con medidas pluviométricas y de temperatura media anual respectivamente de $2.458 \mathrm{~mm} \mathrm{y} 25,6^{\circ} \mathrm{C}$, con una estación seca de junio a octubre (Ribeiro, 1976). En los datos del INMET (Instituto Nacional de Meteorología) referentes a los años de 1961 a 1990 existen registros que muestran una temperatura máxima de $31,5^{\circ} \mathrm{C}$ y mínima de $23,2^{\circ} \mathrm{C}$, temperatura media de $26,7^{\circ} \mathrm{C}$, precipitaciones de $2.291,8 \mathrm{~mm}$ y una humedad relativa del aire de $83 \%$.

En ambos meliponarios, fueron recolectadas semillas dentro y fuera de las colmenas para conmensuración del tamaño (largura y anchura) y posterior plantación de las mismas en vasos desechables de $300 \mathrm{ml}$ y en semilleros de 128 células. Como sustrato fue utilizado el humus colado de gusano. El desarrollo de las plántulas fue acompañado y documentado fotográficamente para servir de comparación con plántulas encontradas en el campo y auxiliar en la identificación de las plantas en cuestión. Las mismas observaciones fueron realizadas en plántulas cercanas a las colmenas.
La actividad de transporte de semillas para dentro y para fuera de las colmenas fue registrada por medio de fotografía digital y filme. Durante el período del estudio, se realizaron seis observaciones en el campo buscando árboles en fase reproductiva (con frutos) para comparación de las semillas de las mismas con aquellas recolectadas en las proximidades e interior de las colmenas. En esas actividades de campo no fueron realizadas observaciones por muestra siendo que dos equipos (con 2 o 3 personas) hacían el sondeo del área, con auxilio de binóculos, en sentidos opuestos para descubrir plantas portadoras de las semillas en cuestión. Los frutos de diversas especies botánicas, cuando encontrados, eran analizados para constatar la presencia de resinas y semillas morfológicamente semejantes a la semilla blanco del estudio. También fue utilizada, por comparación, la literatura de Lorenzi (1998a y b) y Ribeiro et al. (1999) que presentan datos relativos a la fenología, características botánicas y fotografías de frutos y semillas de diferentes especies arbóreas brasileras. La semejanza entre las plántulas presentes cerca de las cajas en los meliponarios y aquellas existentes en las proximidades de la planta madre, observada en el campo, fue constatada también por comparación entre los individuos. Tales observaciones fueron realizadas en un radio de aproximadamente $3 \mathrm{Km}$ de distancia - valor máximo estimado de vuelo para Melipona según Kerr (1996) - para documentarse frutos con las semillas en cuestión o aún la presencia de plántulas semejantes a las obtenidas con las semillas recolectadas.

En esa área de rastreo, se incluyó toda el área del Campus del INPA, sus adyacencias (vegetación de los canteros presentes en el área urbana) y regiones próximas a los senderos y carreteras principales del fragmento forestal urbano de la Universidad Federal del Amazonas - UFAM.

Una filmadora fue instalada, con auxilio de un trípode, adelante de una colonia de $M$. compressipes manaosensis en el meliponario del GPA-INPA. Durante 60 minutos fue monitoreado el flujo de las obreras en esta colonia.

Tras el descubrimiento de dos ejemplares adultos de $Z$. racemosa (distantes aproximadamente $2.150 \mathrm{~m}$ del meliponario GPA-INPA), en la sede de la UFAM, se procedió a una escalada en dosel por medio de elevación vertical para llegar a la copa del árbol y iniciar el registro del comportamiento de la recolección de las semillas hecho por las obreras. Las observaciones de comportamiento realizadas en el dossier de esos dos árboles fueron realizadas en dos días. En el primer árbol, las observaciones duraron 30 minutos y en el segundo árbol, distante $50 \mathrm{~m}$ del primero, las observaciones duraron aproximadamente 2 horas (17:00 a las 19:00 horas por el horario de Brasilia). Una muestra de la planta conteniendo los frutos fue depositada en el herbario del INPA (Bacelar-Lima, C. G. 43, Voucher $\mathrm{n}^{\circ}$ : 215335). 


\section{RESULTADOS Y DISCUSIÓN}

De las veinticinco colmenas presentes en el meliponario Vale Verde, 11 pertenecían a $M$. seminigra merrillae y 13 a $M$. compressipes manaosensis. Fueron encontradas semillas de $Z$. racemosa cerca de las cajas y también en el interior de las colonias de ambas especies de abejas, pudiendo estas, estar incorporadas al geoprópolis o en las fisuras, entre la alza de miel y la tapa. Sin embargo fueron observadas solamente obreras de $M$. compressipes llegando del campo con semillas sujetas a las corbículas (Fig. 2).

De 93 colonias presentes en el meliponario del GPA/INPA, siendo 50 de $M$. seminigra merrillae y 43 de $M$. compressipes manaosensis, 17 colmenas presentaron actividad de recolecta de las semillas de $Z$. racemosa. Fueron recolectadas 80 semillas en la entrada y en las proximidades de las colmenas (Fig. 3) para conmensuración del tamaño (largo y ancho) y posterior plantación. Las larguras (ápice a la base) de las semillas presentaron valor medio de 4,44 $\pm 0,57 \mathrm{~mm}$ y ancho medio de $3,89 \pm 0,56$ $\mathrm{mm}$, representando $32,8 \%$ de la largura corporal de $M$. compressipes manaosensis $(13,5 \pm 0,67 \mathrm{~mm})$ y $37 \%$ de $M$. seminigra merrillae $(12 \pm 0,77 \mathrm{~mm})$. Tan solo dos semillas presentaron resina adherida a la superficie, pero con aspecto reseco no presentando ninguna capacidad adhesiva.

Durante las observaciones de campo fue posible hacer los siguientes registros:

a) Obreras con semillas adheridas, por medio de resina, a las corbículas entrando en la colmena. Sin embargo, se supone que la resina se seca rápidamente, permitiendo la caída de la semilla aun durante el vuelo. Se notó la presencia de varias semillas y de plántulas cercanas a las entradas de las colmenas y fueron encontradas 170 plántulas de $Z$. racemosa en las cercanías del INPA. Las plántulas más lejanas encontradas en los meliponarios del INPA y en el meliponario Vale Verde estaban, respectivamente, a $14 \mathrm{~m}$ y $18 \mathrm{~m}$ de las colmenas. Ya para el meliponario Vale Verde, se encontraron 160 plántulas en las mismas condiciones.

b) Obreras con semillas en las mandíbulas saliendo de las colmenas (desechando las semillas). Inclusive fue constatado, en dos observaciones, que dos semillas que fueron desechadas estaban en proceso de germinación. Con auxilio del filme, fue posible registrar el momento en que una obrera expulsó una semilla del interior de la colonia, transportándola por medio de su mandíbula. Tanto este desecho como la caída de las semillas de las corbículas durante el vuelo pueden indicar la dispersión de esta especie de planta por las especies de M. compressipes manaosensis y M. seminigra merrillae. El desecho de las semillas fue observado en los períodos de la mañana y de la tarde.

c) En relación a las observaciones realizadas en el dossier fue constatada la presencia de frutos maduros y también abejas (Trigonina y Meliponina) recolectando semillas y resina en los dos árboles observados. M. compressipes manaosensis y por lo
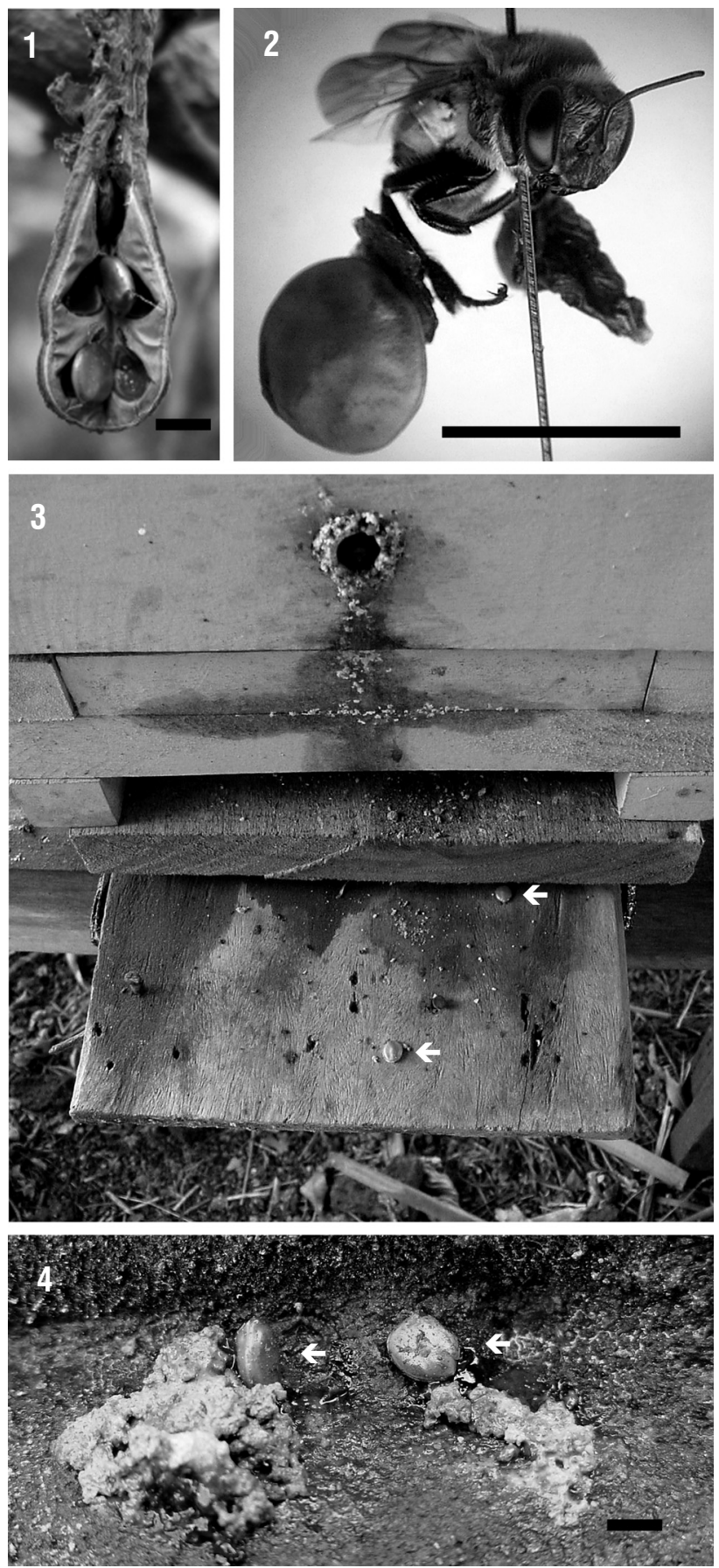

Figuras 1 - Fruto de Zygia racemosa recién abierto exponiendo dos semillas envueltas por resina (barras de escala $=5 \mathrm{~cm}$ ). 2) Melipona compressipes manaosensis capturada y fija con alfileres entomológicos tras intento de entrada en la colmena con dos semillas de Zygia racemosa. 3) Semillas de Zygia racemosa dejadas por las obreras en la puerta de la entrada de una colmena de Melipona compressipes manaosensis, durante el intento de entrada en la colonia transportándolas en las corbículas. 4) Semillas de Zygia racemosa recién mezcladas al barro y resinas (geoprópolis) presente en el basurero de una colmena de Melipona compressipes manaosensis. 
menos otras dos especies representantes del grupo Trigonina (no recolectadas y/o determinadas) fueron observadas recolectando resinas en el interior de las cápsulas de $Z$. racemosa en los dos árboles observados. Sin embargo, apenas $M$. compressipes manaosensis fue observada recolectando no sólo resina, sino también las semillas. Fueron registradas por lo menos 10 obreras de $M$. compressipes manaosensis en el primer árbol y en el segundo, solamente 4 obreras, probablemente debido al horario de las observaciones. A pesar de no haber cuantificación de las abejas Trigonina en los dos árboles observados, por el comparación visual, se constató un mayor número de esas abejas en el relación a Meliponina.

d) En colonias de ambas especies fueron encontradas semillas en los basureros de las colmenas (Fig. 4); mezcladas al geoprópolis y también en las fisuras en las paredes de las colmenas.

García et al. (1992) y Oliveira (2001) aventaron la posibilidad de que estas abejas estuviesen funcionando como agentes de dispersión de semillas de Coussapoa asperifolia. En este estudio, las observaciones en campo también indican la posibilidad de dispersión de semillas por dos especies de Melipona para una importante especie arbórea de la economía regional amazónica $Z y$ gia racemosa. Este fenómeno observado para $Z$. racemosa y para otras especies vegetales (Absy \& Kerr, 1977; Roubik, 1983b y 1989; Garcia et al., 1992; Wallace \& Trueman, 1995; Oliveira, 2001) parece estar íntimamente asociado con la producción de frutos resinosos.

La dispersión de semillas por abejas sin aguijón, también conocida como melitocoria, puede ser considerada como un nuevo y valioso servicio para el mantenimiento de las florestas.

\section{CONCLUSIONES}

Los resultados aquí presentados indican que:

Semillas de Zygia racemosa, popularmente conocido como "anjelim rajado", son recolectadas por las especies de abejas sin aguijón $M$. compressipes manaosensis y M. seminigra merrillae.

Las semillas pueden ser aprovechadas, en el interior de las colonias de esas abejas, como material de construcción incorporado al geoprópolis, mientras otras son desechadas.

$M$. compressipes manaosensis y $M$. seminigra merrillae constituyen agentes dispersores de semillas de Zygia racemosa, promoviendo la propagación de plántulas cerca de los meliponarios, en fragmentos o en la propia floresta.

Obreras de $M$. compressipes manaosensis pueden recolectan solo resina durante las visitas a la $Z$. racemosa.

Las semillas pueden ser dispersadas de dos formas: caída de las semillas atrapadas en las corbículas durante el vuelo y desecho de las semillas previamente en el interior de las colonias.

\section{AGRADECIMIENTOS}

Los autores agradecen al CNPq, SUFRAMA y al IBAMA/ Provarzea por el auxilio concedido y las becas de estudio. Al INPA, a CPCA/GPA por el apoyo logístico y a la UFAM por permitir las observaciones en la sede universitaria (fragmento forestal). Agradecen también al Dr. Kaoru Yuyama (INPA/CPCA) por viabilizar este estudio en su meliponario experimental - Vale Verde.

\section{REFERÊNCIAS BIBLIOGRÁFICAS}

Absy, M.L.; Kerr, W.E. 1977. Algumas plantas visitadas para obtenção de pólen por operárias de Melipona seminigra merrillae em Manaus. Acta Amazonica 7: 309-315.

Alencar, J.C. 1998. Fenologia de espécies arbóreas tropicais na Amazônia Central. In: Gascon, C.; Montinho, P. (eds). Floresta Amazônica: Dinâmica, Regeneração e Manejo. INPA. Manaus, Amazonas. 373pp.

Ferraz, I.D.K.; Filho, N.L.; Imakawa, M.A.; Varela, V.P.; PiñaRodrigues, F.C.M. 2004. Características básicas para um agrupamento ecológico preliminar de espécies madeireiras da floresta de terra firme da Amazônia Central. Acta Amazonica 34(4): $621-633$.

Ferreira, C.A.C.; J. F. Ramos. 1993. Espécies botânicas do Campus do INPA: arbustivas e arbóreas. INPA: Manaus-AM. 17pp.

Ferreira, G.C.; Martins-da-Silva, R.C.V.; Gomes, J.I. 2001. Contribuição ao Conhecimento de Espécies de Leguminosae, Conhecidas na Amazônia Brasileira como "Angelim" e Variações. In: Silva, J.N.M.; Carvalho, J.O.P.; Yared, J.A.G. (Eds). A Silvicultura na Amazônia Oriental-Contribuiçôes do Projeto Embrapa/DFID: Belém. 459pp.

Fonseca, C.R.V.; Neves, R.S.; Ferreira, E.J.G.; Oliveira, L.A.; Filho, A.O.M.; Magnusson, W.E. 1991. Catálogo de Madeiras da Amazônia: Características tecnológicas; Área da Hidrelétrica de Balbina. INPA/CPPF. Manaus. 163pp.

Garcia, M.V.B.; Oliveira, M.L.; Campos, A.O. 1992. Use of Seeds of Coussapoa asperifolia magnifolia (Cecropiaceae) by Stingles Bees in the Central Amazonian Forest (Hymenoptera: Apidae: Meliponinae). Entomologia Generalis 17(4): 255-258.

Gentry, A.H. 1978. Diversidade e regeneração da capoeira do INPA, com referência especial as Bignoniaceae. Acta Amazonica 8 (1): 67-70.

Ihering, H. Von. 1903a. Biolgie der stachellosen Honigbienen Brasiliens. Zoologische Jahrbuecher Abteilung fuer Systematik Oekologie und Geographie der Tiere 19: 179-287.

Ihering, H. Von. 1903b. Biologia das abelhas melíferas do Brasil. Boletim da. Secretaria de Agricultura do Estado de São Paulo 31: 435-506.

Kerr, W.E. 1996. Biologia e Manejo da Tiúba: a abelha do Maranhão. EDUFMA. São Luiz, MA. 165pp. 


\section{ACTA AMAZONICA}

Kerr, W. E.; Carvalho, G. A.; Nascimento, V.A. 1999. The Probable Consequences of The Destruction of Brazilian Stingless Bess In: Padoch, C.; Ayres, J.M.; Pinedo-Vasquez, M.; Henderson, A. (eds). Várzea: diversity, development, and conservation of Amazonia's whitewater floodplains, The New York Botanical Garden Press, Advances in Economic Botany, 3: 395-403.

Kerr, W. E.; Carvalho, G. A.; Coletto-Silva, A.; Assis, M.G.P. 2001. Aspectos Pouco Mencionados da Biodiversidade Amazônica, Parcerias Estratégicas, Ministério da Ciência e Tecnologia 12: 20-41.

Lima-Filho, D.A.; Revilla, J.; Amaral, I.L.; Matos, F.D.A.; Coelho, L.S.; Ramos, J.F.; Silva, G. B.; Guedes, J.O. 2004. Aspectos florísticos de 13 hectares da área de Cachoeira Porteira-PA. Acta Amazonica 34(3): 365-379.

Lorenzi, H. 1998a. Arvores Brasileiras, Manual de Identificação e Cultivo de Plantas Arbóreas do Brasil. 2a ed., Editora Plantarum, Nova Odessa - SP, volume 1, 368p.

Lorenzi, H. 1998b. Arvores Brasileiras, Manual de Identificação e Cultivo de Plantas Arbóreas do Brasil. Nova Odessa - SP, Editora Plantarum, volume 2, 368p.

Michener, C.D. 1974. The Social Behavior of The Bees: A Comparative Study. Cambridge, Massachusetts: Harvard University Press, ixii, 404pp.

Nogueira-Neto, P. 1997. Vida e criação de Abelhas Indigenas Sem Ferrão. São Paulo - SP, Editora Nogueirapis, Brasil, 445pp.

Oliveira, M.L. 2001. Stingless Bees (Meliponini) and Orchid bees (Euglossini) in Terra Firme, Tropical Forests and Forest Fragments. In: Bierregaard Jr., R.O., Gascon, C.; Lovejoy, T.E. \& Mesquita, R. (Eds.). Lessons from Amazonia, The Ecology and Conservation of a Fragmented Forest. Yale University Press, New Haven, EUA, 496pp.
Prance, G.T. 1975. The history of the INPA capoeira based on ecological studies of Lecythidaceae. Acta Amazonica 5 (3): 261263.

Ribeiro, J.E.L.S.; Hopkins, M.J.G.; Vicentine, A.; Sothers, C.A.; Costa, M.A.S.; Brito, J.M.; Souza, M.A.D.; Martins, L.H.P.; Lohmann, L.G.; Assunção, P.A.C.L.; Pereira, E.C.; Silva, C.F.; Mesquita, M.R.; Procópio, L.C. 1999. Flora da Reserva Ducke: Guia de identificação das Plantas Vasculares de uma Floresta de Terra Firme na Amazônia Central. INPA, Manaus, Amazonas. 816pp.

Ribeiro, M.N.G. 1976. Aspectos climatológicos de Manaus. Acta Amazonica, 6 (2): 229-233.

Roubik, D.W.1983a. Nest and colony characteristics of stingless bees from Panamá. Journal of Kansas Entomology Society 56: 327 355.

Roubik, D.W.1983b. Experimental community studies: time-series tests of competition between African and neotropical bees. Ecology 64: 971-978.

Roubik, D. W. 1989 - Ecology and Natural History of Tropical Bees, Cambridge Tropical Biology Series, Cambridge University Press, Cambridge, New York, 514pp.

Silveira, F.A.; Melo, G.A.R.; Almeida, E.A.B. 2002. Abelhas Brasileiras: sistemática e identificação. Ed. MMA e Fundação Araucária, Curitiba-PR, $1^{\circ}$ ed. $253 \mathrm{pp}$.

Wallace, H. M.; Trueman, S. J. 1995. Dispersal of Eucalyptus torelliana seeds by the resin-collecting stingless bee, Trigona carbonaria. Oecologia, 104: 12-16.

Recebido em 19/05/2005

Aceito em 25/07/2006 